\title{
A LÊ-GREUEL TYPE FORMULA FOR THE IMAGE MILNOR NUMBER
}

\author{
J.J. NUÑO-BALLESTEROS, I. PALLARÉS-TORRES
}

\begin{abstract}
Let $f:\left(\mathbb{C}^{n}, 0\right) \rightarrow\left(\mathbb{C}^{n+1}, 0\right)$ be a corank 1 finitely determined map germ. For a generic linear form $p:\left(\mathbb{C}^{n+1}, 0\right) \rightarrow(\mathbb{C}, 0)$ we denote by $g:\left(\mathbb{C}^{n-1}, 0\right) \rightarrow\left(\mathbb{C}^{n}, 0\right)$ the transverse slice of $f$ with respect to $p$. We prove that the sum of the image Milnor numbers $\mu_{I}(f)+\mu_{I}(g)$ is equal to the number of critical points of $\left.p\right|_{X_{s}}: X_{s} \rightarrow \mathbb{C}$ on all the strata of $X_{s}$, where $X_{s}$ is the disentanglement of $f$ (i.e., the image of a stabilisation $f_{s}$ of $f$ ).
\end{abstract}

\section{INTRODUCTION}

The Lê-Greuel formula $[4,6]$ provides a recursive method to compute the Milnor number of an isolated complete intersection singularity (ICIS). We recall that if $(X, 0)$ is a $d$-dimensional ICIS defined as the zero locus of a map germ $g:\left(\mathbb{C}^{n}, 0\right) \rightarrow\left(\mathbb{C}^{n-d}, 0\right)$, then the Milnor fibre $X_{s}=g^{-1}(s)$ (where $s$ is a generic value in $\mathbb{C}^{n-d}$ ) has the homotopy type of a bouquet of $d$-spheres and the number of such spheres is called the Milnor number $\mu(X, 0)$. If $d>0$, we can take $p: \mathbb{C}^{n} \rightarrow \mathbb{C}$ a generic linear projection with $H=p^{-1}(0)$ and such that $(X \cap H, 0)$ is a $(d-1)$-dimensional ICIS. Then,

$$
\mu(X, 0)+\mu(X \cap H, 0)=\operatorname{dim}_{\mathbb{C}} \frac{\mathcal{O}_{n}}{(g)+J(g, p)},
$$

where $\mathcal{O}_{n}$ is the ring of function germs from $\left(\mathbb{C}^{n}, 0\right)$ to $\mathbb{C},(g)$ is the ideal in $\mathcal{O}_{n}$ generated by the components of $g$ and $J(g, p)$ is the Jacobian ideal of $(g, p)$ (i.e., the ideal generated by the maximal minors of the Jacobian matrix). Note that $X_{s}$ is smooth and if $p$ is generic enough, then the restriction $\left.p\right|_{X_{s}}: X_{s} \rightarrow \mathbb{C}$ is a Morse function and the dimension appearing in the right hand side of (1) is equal to the number of critical points of $\left.p\right|_{X_{s}}$.

The aim of this paper is to obtain a Lê-Greuel type formula for the image Milnor number of a finitely determined map germ $f:\left(\mathbb{C}^{n}, 0\right) \rightarrow\left(\mathbb{C}^{n+1}, 0\right)$. Mond showed in [11] that the disentanglement $X_{s}$ (i.e., the image of a stabilisation $f_{s}$ of $f$ ) has the homotopy type of a bouquet of $n$-spheres and the number of such spheres is called the image Milnor number $\mu_{I}(f, 0)$. The celebrated Mond's conjecture says that

$$
\mathcal{A}_{e^{-}} \operatorname{codim}(f) \leq \mu_{I}(f),
$$

with equality if $f$ is weighted homogeneous. Mond's conjecture is known to be true for $n=1,2$ but it remains still open for $n \geq 3$ (see [11, 12]). We feel that our Lê-Greuel type formula can be useful to find a proof of the

2000 Mathematics Subject Classification. Primary 32S30; Secondary 32S05, 58K40.

Key words and phrases. Image Milnor number, Lê-Greuel formula, finite determinacy. Work partially supported by DGICYT Grant MTM2015-64013-P. 
conjecture in the general case. In fact, it would be enough to prove that the module which controls the number of critical points of a generic linear function is Cohen-Macaulay and then, use an induction argument on the dimension $n$ (see [1] for details about Mond's conjecture).

We assume that $f$ has corank 1 and $n>1$. Then given a generic linear form $p: \mathbb{C}^{n+1} \rightarrow \mathbb{C}$ we can see $f$ as a 1-parameter unfolding of another map germ $g:\left(\mathbb{C}^{n-1}, 0\right) \rightarrow\left(\mathbb{C}^{n}, 0\right)$ which is the transverse slice of $f$ with respect to $p$. This means that $g$ has image $(X \cap H, 0)$, where $(X, 0)$ is the image of $f$ and $H=p^{-1}(0)$. The disentanglement $X_{s}$ is not smooth but it has a natural Whitney stratification given by the stable types. If $p$ is generic enough, the restriction $\left.p\right|_{X_{s}}: X_{s} \rightarrow \mathbb{C}$ is a Morse function on each stratum. Our Lê-Greuel type formula is

$$
\mu_{I}(f)+\mu_{I}(g)=\# \Sigma\left(\left.p\right|_{X_{s}}\right),
$$

where the right hand side of equation is the number of critical points of $\left.p\right|_{X_{s}}$ on all the strata of $X_{s}$. The case $n=1$ has to be considered separately, in this case we have

$$
\mu_{I}(f)+m_{0}(f)-1=\# \Sigma\left(\left.p\right|_{X_{s}}\right),
$$

where $m_{0}(f)$ is the multiplicity of the curve parametrized by $f$. This makes sense, since $\mu(X, 0)=m_{0}(X, 0)-1$ for a 0 -dimensional ICIS $(X, 0)$.

\section{Multiple point spaces and Marar's formula}

In this section we recall Marar's formula for the Euler characteristic of the disentanglement of a corank 1 finitely determined map germ. We first recall the Marar-Mond [9] construction of the $k$ th-multiple point spaces for corank 1 map germs, which is based on the iterated divided differences. Let $f:\left(\mathbb{C}^{n}, 0\right) \rightarrow\left(\mathbb{C}^{p}, 0\right)$ be a corank 1 map germ. We can choose coordinates in the source and target such that $f$ is written in the following form:

$$
f(x, z)=\left(x, f_{n}(x, z), \ldots, f_{p}(x, z)\right), x \in \mathbb{C}^{n-1}, z \in \mathbb{C} .
$$

This forces that if $f\left(x_{1}, z_{1}\right)=f\left(x_{2}, z_{2}\right)$ then necessarily $x_{1}=x_{2}$. Thus, it makes sense to embed the double point space of $f$ in $\mathbb{C}^{n-1} \times \mathbb{C}^{2}$ instead of $\mathbb{C}^{n} \times \mathbb{C}^{n}$. Analogously, we will consider the $k$ th-multiple point space embedded in $\mathbb{C}^{n-1} \times \mathbb{C}^{k}$.

We construct an ideal $I_{k}(f) \subset \mathcal{O}_{n+k-1}$ defined as follows: $I_{k}(f)$ is generated by $(k-1)(p-n+1)$ functions $\Delta_{i}^{(j)} \in \mathcal{O}_{n+k-1}, 1 \leq i \leq k-1, n \leq j \leq p$. Each $\Delta_{i}^{(j)}$ is a function only of the variables $x, z_{1}, \ldots, z_{i+1}$ such that:

$$
\Delta_{1}^{(j)}\left(x, z_{1}, z_{2}\right)=\frac{f_{j}\left(x, z_{1}\right)-f_{j}\left(x, z_{2}\right)}{z_{1}-z_{2}}
$$

and for $1 \leq i \leq k-2$,

$$
\Delta_{i+1}^{(j)}\left(x, z_{1}, \ldots, z_{i+2}\right)=\frac{\Delta_{i}^{(j)}\left(x, z_{1}, \ldots, z_{i}, z_{i+1}\right)-\Delta_{i}^{(j)}\left(x, z_{1}, \ldots, z_{i}, z_{i+2}\right)}{z_{i+1}-z_{i+2}} .
$$

Definition 2.1. The $k$ th-multiple point space is $D^{k}(f)=V\left(I_{k}(f)\right)$, the zero locus in $\left(\mathbb{C}^{n+k-1}, 0\right)$ of the ideal $I_{k}(f)$. 
(We remark that the $k$ th-multiple point space is denoted by $\widetilde{D}^{k}(f)$ instead of $D^{k}(f)$ in [9]).

If $f$ is stable, then, set-theoretically, $D^{k}(f)$ is the Zariski closure of the set of points $\left(x, z_{1}, \ldots, z_{k}\right) \in \mathbb{C}^{n+k-1}$ such that:

$$
f\left(x, z_{1}\right)=\ldots=f\left(x, z_{k}\right), \quad z_{i} \neq z_{j}, \text { for } i \neq j,
$$

(see $[9,13])$. But, in general, this may be not true if $f$ is not stable. For instance, consider the cusp $f:(\mathbb{C}, 0) \rightarrow\left(\mathbb{C}^{2}, 0\right)$ given by $f(z)=\left(z^{2}, z^{3}\right)$. Since $f$ is one-to-one, the closure of the double point set is empty, but

$$
D^{2}(f)=V\left(z_{1}+z_{2}, z_{1}^{2}+z_{1} z_{2}+z_{2}^{2}\right) .
$$

This example also shows that the $k$ th-multiple point space may be nonreduced in general.

The main result of Marar-Mond in [9] is that the $k$ th-multiple point spaces can be used to characterize the stability and the finite determinacy of $f$.

Theorem 2.2. $[9,2.12]$ Let $f:\left(\mathbb{C}^{n}, 0\right) \rightarrow\left(\mathbb{C}^{p}, 0\right)(n<p)$ be a finitely determined map germ of corank 1. Then:

(1) $f$ is stable if and only if $D^{k}(f)$ is smooth of dimension $p-k(p-n)$, or empty, for $k \geq 2$.

(2) $f$ is finitely determined if and only if for each $k$ with $p-k(p-n) \geq 0$, $D^{k}(f)$ is either an ICIS of dimension $p-k(p-n)$ or empty, and if, for those $k$ such that $p-k(p-n)<0, D^{k}(f)$ consists at most of the point $\{0\}$.

The following construction is also due to Marar-Mond [9] and gives a refinement of the types of multiple points.

Definition 2.3. Let $\mathcal{P}=\left(r_{1}, \ldots, r_{m}\right)$ be a partition of $k$ (that is, $r_{1}+\ldots+$ $r_{m}=k$, with $\left.r_{1} \geq \cdots \geq r_{m}\right)$. Let $I(\mathcal{P})$ be the ideal in $\mathcal{O}_{n-1+k}$ generated by the $k-m$ elements $z_{i}-z_{i+1}$ for $r_{1}+\ldots+r_{j-1}+1 \leq i \leq r_{1}+\ldots+r_{j}$ for $j=1, \ldots, m$. Define the ideal $I_{k}(f, \mathcal{P})=I_{k}(f)+I(\mathcal{P})$ and the $k$-multiple point space of $f$ with respect to the partition $\mathcal{P}$ as $D^{k}(f, \mathcal{P})=V\left(I_{k}(f, \mathcal{P})\right)$.

Definition 2.4. We define a generic point of $D^{k}(f, \mathcal{P})$ as a point

$$
\left(x, z_{1}, \ldots, z_{1}, \ldots, z_{m}, \ldots, z_{m}\right),
$$

$\left(z_{i}\right.$ iterated $r_{i}$ times, and $z_{i} \neq z_{j}$ if $i \neq j$ ) such that the local algebra of $f$ at $\left(x, z_{i}\right)$ is isomorphic to $\mathbb{C}[t] /\left(t^{r_{i}}\right)$, and such that

$$
f\left(x, z_{1}\right)=\ldots=f\left(x, z_{m}\right) .
$$

If $f$ is stable, then $D^{k}(f, \mathcal{P})$ is equal to the Zariski closure of its generic points (see [9]). Moreover, we have the following corollary, which extends Theorem 2.2 to the multiple point spaces with respect to the partitions.

Corollary 2.5. [9, 2.15] If $f$ is finitely determined (resp. stable), then for each partition $\mathcal{P}=\left(r_{1}, \ldots, r_{m}\right)$ of $k$ satisfying $p-k(p-n+1)+m \geq 0$, the germ of $D^{k}(f, \mathcal{P})$ at $\{0\}$ is either an ICIS (resp. smooth) of dimension $p-k(p-n+1)+m$, or empty. Moreover, those $D^{k}(f, \mathcal{P})$ for $\mathcal{P}$ not satisfying the inequality consist at most of the single point $\{0\}$. 
Let $f:\left(\mathbb{C}^{p}, 0\right) \rightarrow\left(\mathbb{C}^{p}, 0\right)$ be a finitely determined map germ of corank 1 and let $f_{s}: U_{s} \rightarrow X_{s}$ be a stabilization of $f$. For a partition $\mathcal{P}$ of $k$, we denote by $\rho_{\mathcal{P}}$ the mapping given as the composition of the inclusion $D^{k}\left(f_{s}, \mathcal{P}\right) \hookrightarrow D^{k}\left(f_{s}\right)$, the projection $D^{k}\left(f_{s}\right) \rightarrow U_{s}$ and $f_{s}$. The following two results will be useful in the next section.

Remark 2.6. [8] Let $\mathcal{P}=\left(a_{1}, \ldots, a_{h}\right)$ be a partition of $k$, with $a_{i} \geq a_{i+1}$. If $y$ is a generic point of $D^{k}\left(f_{s}, \mathcal{P}^{\prime}\right)$, where $\mathcal{P}^{\prime}=\left(b_{1}, \ldots, b_{q}\right)$, with $b_{i} \geq b_{i+1}$ and $\mathcal{P}<\mathcal{P}^{\prime}$ then $\# \rho_{\mathcal{P}}^{-1}\left(\rho_{\mathcal{P}^{\prime}}(y)\right)$ is the coefficient of the monomial $x_{1}^{b_{1}} x_{2}^{b_{2}} \ldots x_{q}^{b_{q}}$ in the polynomial $\prod_{i \geq 1}\left(x_{1}^{a_{i}}+x_{2}^{a_{i}}+\ldots x_{q}^{a_{i}}\right)$.

Lemma 2.7. [7] Let $h_{k}$ be the $k$-th complete symmetric function in variables $x_{1}, \ldots, x_{q}$, i.e., $h_{k}$ is the sum of all monomials of degree $k$ in the variables $x_{1}, \ldots, x_{q}$. Then

$$
h_{k}=\sum_{\mathcal{P}} \frac{1}{\prod_{i \geq 1} \alpha_{i} ! i^{\alpha_{i}}} \prod_{i \geq 1}\left(x_{1}^{i}+\ldots+x_{q}^{i}\right)^{\alpha_{i}},
$$

where $\mathcal{P}$ runs through the set of all ordered partitions of $k$.

The next step is to observe that the $k$ th-multiple point space $D^{k}(f)$ is invariant under the action of the $k$ th symmetric group $S_{k}$.

Definition 2.8. Let $M$ be a $\mathbb{Q}$-vector space upon which $S_{k}$ acts. Then the alternating part of $M$, denoted by $\operatorname{Alt}_{k} M$, is defined to be

$$
\operatorname{Alt}_{k} M:=\left\{m \in M: \sigma(m)=\operatorname{sign}(\sigma) m, \text { for all } \sigma \in S_{k}\right\} .
$$

Given a topological space $X$ on which $S_{k}$ acts, the alternating Euler characteristic is

$$
\chi^{\text {alt }}(X):=\sum_{i}(-1)^{i} \operatorname{dim}_{\mathbb{Q}} \operatorname{Alt}_{k}\left(H_{i}(X, \mathbb{Q})\right) .
$$

The following theorem of Goryunov-Mond in [3] allows us to compute the image Milnor number of $f$ by means of a spectral sequence associated to the multiple point spaces.

Theorem 2.9. $[3,2.6]$ Let $f:\left(\mathbb{C}^{n}, 0\right) \rightarrow\left(\mathbb{C}^{n+1}, 0\right)$ be a corank 1 map germ and $f_{s}$ a stabilisation of $f$, for $s \neq 0$ and $X_{s}$ the image of $f_{s}$. Then,

$$
H_{n}\left(X_{s}, \mathbb{Q}\right) \cong \bigoplus_{k=2}^{n+1} \operatorname{Alt}_{k}\left(H_{n-k+1}\left(D^{k}\left(f_{s}\right), \mathbb{Q}\right)\right)
$$

Note that since $X_{s}$ has the homotopy type of a wedge of $n$-spheres, the image Milnor number of $f$ is the rank of $H_{n}\left(X_{s}, \mathbb{Q}\right)$. If we consider $H_{n}\left(X_{s}, \mathbb{Q}\right)$ as a $\mathbb{Q}$-vector space,

$$
\mu_{I}(f)=\operatorname{dim}_{\mathbb{Q}} H_{n}\left(X_{s}, \mathbb{Q}\right) .
$$

So, by Theorem 2.9, the image Milnor number is

$$
\mu_{I}(f)=\sum_{k=2}^{n+1} \operatorname{dim}_{\mathbb{Q}} \operatorname{Alt}_{k}\left(H_{n-k+1}\left(D^{k}\left(f_{s}\right), \mathbb{Q}\right)\right) .
$$


By [5, Corollary 2.8], we can compute the alternating Euler characteristic of $D^{k}\left(f_{s}\right)$ as follows: for each partition $\mathcal{P}=\left(r_{1}, \ldots, r_{s}\right)$, we set

$$
\beta(\mathcal{P})=\frac{\operatorname{sign}(\mathcal{P})}{\prod_{i} \alpha_{i} ! i^{\alpha_{i}}},
$$

where $\alpha_{i}:=\#\left\{j: r_{j}=i\right\}$ and $\operatorname{sign}(\mathcal{P})$ is the number $(-1)^{k-\sum_{i} \alpha_{i}}$. Then,

$$
\chi^{\text {alt }}\left(D^{k}\left(f_{s}\right)\right)=\sum_{|\mathcal{P}|=k} \beta(\mathcal{P}) \chi\left(D^{k}\left(f_{s}, \mathcal{P}\right)\right) .
$$

Moreover, by Theorem 2.2 and Corollary $2.5, D^{k}\left(f_{s}\right)\left(\right.$ resp. $D^{k}\left(f_{s}, \mathcal{P}\right)$ ) is a Milnor fibre of the ICIS $D^{k}(f)$ (resp. $D^{k}(f, \mathcal{P})$ ), and hence it has the homotopy type of a wedge of spheres of real dimension $\operatorname{dim} D^{k}(f)=n-k+1$ (resp. $\left.\operatorname{dim} D^{k}(f, \mathcal{P})\right)$. Thus,

$$
\operatorname{dim}_{\mathbb{Q}} \operatorname{Alt}_{k}\left(H_{n-k+1}\left(D^{k}\left(f_{s}\right), \mathbb{Q}\right)\right)=(-1)^{n-k+1} \chi^{a l t}\left(D^{k}\left(f_{s}\right)\right),
$$

and

$$
\chi\left(D^{k}\left(f_{s}, \mathcal{P}\right)\right)=1+(-1)^{\operatorname{dim} D^{k}(f, \mathcal{P})} \mu\left(D^{k}(f, \mathcal{P})\right) .
$$

This gives the following version of Marar's formula [8] in terms of the Milnor numbers of the multiple point spaces:

$$
\mu_{I}(f)=\sum_{k=2}^{n+1}(-1)^{n-k+1} \sum_{|\mathcal{P}|=k} \beta(\mathcal{P})\left(1+(-1)^{\operatorname{dim} D^{k}(f, \mathcal{P})} \mu\left(D^{k}(f, \mathcal{P})\right)\right),
$$

where the coefficients $\beta(\mathcal{P})=0$ when the sets $D^{k}(f, \mathcal{P})$ are empty, for $k=2, \ldots, n+1$.

\section{LÊ-GREuEl TyPe FORMUlA}

Let $f:\left(\mathbb{C}^{n}, 0\right) \rightarrow\left(\mathbb{C}^{n+1}, 0\right)$ be a corank 1 finitely determined map germ. Let $p: \mathbb{C}^{n+1} \rightarrow \mathbb{C}$ be a generic linear projection such that $H=p^{-1}(0)$ is a generic hyperplane through the origin in $\mathbb{C}^{n+1}$. We can choose linear coordinates in $\mathbb{C}^{n+1}$ such that $p\left(y_{1}, \ldots, y_{n+1}\right)=y_{1}$. Then, we choose the coordinates in $\mathbb{C}^{n}$ in such a way that $f$ is written in the form

$$
f\left(x_{1}, \ldots, x_{n-1}, z\right)=\left(x_{1}, \ldots, x_{n-1}, h_{1}\left(x_{1}, \ldots, x_{n-1}, z\right), h_{2}\left(x_{1}, \ldots, x_{n-1}, z\right)\right),
$$

for some holomorphic functions $h_{1}, h_{2}$. We see $f$ as a 1-parameter unfolding of the map germ $g:\left(\mathbb{C}^{n-1}, 0\right) \rightarrow\left(\mathbb{C}^{n}, 0\right)$ given by

$g\left(x_{2}, \ldots, x_{n-1}, z\right)=\left(x_{2}, \ldots, x_{n-1}, h_{1}\left(0, x_{2}, \ldots, x_{n-1}, z\right), h_{2}\left(0, x_{2}, \ldots, x_{n-1}, z\right)\right)$.

We say that $g$ is the transverse slice of $f$ with respect to the generic hyperplane $H$. If $f$ has image $(X, 0)$ in $\left(\mathbb{C}^{n+1}, 0\right)$, then the image of $g$ in $\left(\mathbb{C}^{n}, 0\right)$ is isomorphic to $(X \cap H, 0)$.

We take $f_{s}$ a stabilisation of $f$ and denote by $X_{s}$ the image of $f_{s}$ (see [11] for the definition of stabilisation). Since $f$ has corank $1, X_{s}$ has a natural Whitney stratification given by the stable types of $f_{s}$. In fact, the strata are the submanifolds

$$
M^{k}\left(f_{s}, \mathcal{P}\right):=\epsilon^{k}\left(D^{k}\left(f_{s}, \mathcal{P}\right)^{0}\right) \backslash \epsilon^{k+1}\left(D^{k+1}\left(f_{s}\right)\right),
$$

where $D^{k}\left(f_{s}, \mathcal{P}\right)^{0}$ is the set of generic points of $D^{k}\left(f_{s}, \mathcal{P}\right), \epsilon^{k}: \mathbb{C}^{n+k-1} \rightarrow$ $\mathbb{C}^{n+1}$ is the map $\left(x, z_{1}, \ldots, z_{k}\right) \mapsto f_{s}\left(x, z_{1}\right)$ and $\mathcal{P}$ runs through all the partitions of $k$ with $k=2, \ldots, n+1$. We can choose the generic linear 
projection $p: \mathbb{C}^{n+1} \rightarrow \mathbb{C}$ in such a way that the restriction to each stratum $M^{k}\left(f_{s}, \mathcal{P}\right)$ is a Morse function. In other words, such that the restriction $\left.p\right|_{X_{s}}: X_{s} \rightarrow \mathbb{C}$ is a Morse function on each stratum (this is one of the condition of be a stratifed Morse function in the sense of [2]). We will denote by $\# \Sigma\left(\left.p\right|_{X_{s}}\right)$ the number of critical points on all the strata of $X_{s}$. Our first result in this section is for the case of a plane curve.

Theorem 3.1. Let $f:(\mathbb{C}, 0) \rightarrow\left(\mathbb{C}^{2}, 0\right)$ be an injective map germ. Let $p: \mathbb{C}^{2} \rightarrow \mathbb{C}$ be a generic linear projection, then

$$
\# \Sigma\left(\left.p\right|_{X_{s}}\right)=\mu_{I}(f)+m_{0}(f)-1
$$

where $m_{0}(f)$ is the multiplicity of $f$.

Proof. After a change of coordinates, we can assume that

$$
f(t)=\left(t^{k}, c_{m} t^{m}+c_{m+1} t^{m+1}+\ldots\right),
$$

where $k=m_{0}(f), m>k$ and $c_{m} \neq 0$. The stabilisation $f_{s}$ is an immersion with only transverse double points. So, its image $X_{s}$ has only two strata: $M^{2}\left(f_{s},(1,1)\right)$ is a 0 -dimensional stratum composed by the transverse double points and $M^{1}\left(f_{s},(1)\right)$ is a 1-dimensional stratum given by the smooth points of $X_{s}$. Note that the number of double points of $f_{s}$ is the delta invariant of the plane curve, $\delta(X, 0)$, which is equal to $\mu_{I}(f)$ by [12, Theorem 2.3].

Let $p: \mathbb{C}^{2} \rightarrow \mathbb{C}$ be a generic linear projection such that $\left.p\right|_{X_{s}}$ is a Morse function on each stratum. Then:

$$
\# \Sigma\left(\left.p\right|_{X_{s}}\right)=\# M^{2}\left(f_{s},(1,1)\right)+\# \Sigma\left(\left.p\right|_{M^{1}\left(f_{s},(1)\right)}\right)=\mu_{I}(f)+\# \Sigma\left(\left.p\right|_{M^{1}\left(f_{s},(1)\right)}\right) \text {. }
$$

Since $f_{s}$ is a local diffeomorphism on the stratum $M^{1}\left(f_{s},(1)\right)$, the number of critical points of $\left.p\right|_{M^{1}\left(f_{s},(1)\right)}$ is equal to the number of critical points of $p \circ f_{s}$ (here the points of $M^{2}\left(f_{s},(1,1)\right)$ can be excluded by the genericity of $p$ ). Assume that $p(x, y)=A x+B y$ with $A \neq 0$. Then $p \circ f_{s}$ is a Morsification of the function

$$
p \circ f(t)=A t^{k}+B\left(c_{m} t^{m}+c_{m+1} t^{m+1}+\ldots\right)
$$

The number of critical points of $p \circ f_{s}$ is equal to $\mu(p \circ f)=k-1=m_{0}(f)-1$, which proves our formula.

Next, we state and prove the formula for the case $n>1$.

Theorem 3.2. Let $f:\left(\mathbb{C}^{n}, 0\right) \rightarrow\left(\mathbb{C}^{n+1}, 0\right)$ be a corank 1 finitely determined map germ with $n>1$. Let $p: \mathbb{C}^{n+1} \rightarrow \mathbb{C}$ be a generic linear projection which defines a transverse slice $g:\left(\mathbb{C}^{n-1}, 0\right) \rightarrow\left(\mathbb{C}^{n}, 0\right)$. Then,

$$
\# \Sigma\left(\left.p\right|_{X_{s}}\right)=\mu_{I}(f)+\mu_{I}(g) \text {. }
$$

Proof. By Marar's formula (4):

$$
\begin{aligned}
\mu_{I}(f)+\mu_{I}(g) & =\sum_{k=2}^{n+1}(-1)^{n-k+1} \sum_{|\mathcal{P}|=k} \beta(\mathcal{P})\left(1+(-1)^{\operatorname{dim} D^{k}(f, \mathcal{P})} \mu\left(D^{k}(f, \mathcal{P})\right)\right) \\
& +\sum_{k=2}^{n}(-1)^{n-k} \sum_{|\mathcal{P}|=k} \beta(\mathcal{P})\left(1+(-1)^{\operatorname{dim} D^{k}(g, \mathcal{P})} \mu\left(D^{k}(g, \mathcal{P})\right)\right)
\end{aligned}
$$


Note that if $\operatorname{dim} D^{k}(f, \mathcal{P})>0$, then $\operatorname{dim} D^{k}(f, \mathcal{P})=1+\operatorname{dim} D^{k}(g, \mathcal{P})$. Moreover, if $\operatorname{dim} D^{k}(f, \mathcal{P})=0$, then $D^{k}(g, \mathcal{P})=\emptyset$. So, we can separate the formula into two parts, the first one for partitions with $\operatorname{dim} D^{k}(f, \mathcal{P})=0$, the second one for partitions with $\operatorname{dim} D^{k}(f, \mathcal{P})>0$. Thus,

$$
\begin{aligned}
& \mu_{I}(f)+\mu_{I}(g)=\sum_{k=2}^{n+1}(-1)^{n+k-1} \sum_{\substack{|\mathcal{P}|=k \\
\operatorname{dim} D^{k}(f, \mathcal{P})=0}} \beta(\mathcal{P})\left(1+\mu\left(D^{k}(f, \mathcal{P})\right)\right) \\
& +\sum_{k=2}^{n}(-1)^{n+k-1} \sum_{\substack{|\mathcal{P}|=k \\
\operatorname{dim} D^{k}(f, \mathcal{P})>0}} \beta(\mathcal{P})(-1)^{\operatorname{dim} D^{k}(f, \mathcal{P})}\left(\mu\left(D^{k}(f, \mathcal{P})\right)+\mu\left(D^{k}(g, \mathcal{P})\right)\right)
\end{aligned}
$$

If $\operatorname{dim} D^{k}(f, \mathcal{P})=0$, the Milnor number of $D^{k}(f, \mathcal{P})$ is

$$
\mu\left(D^{k}(f, \mathcal{P})\right)=\operatorname{deg}\left(D^{k}(f, \mathcal{P})\right)-1,
$$

where $d e g$ is the degree of the map germ that defines the 0-dimensional ICIS $D^{k}(f, \mathcal{P})$. Note that we can see $\operatorname{deg}\left(D^{k}(f, \mathcal{P})\right)$ as the number of critical points of $\left.\tilde{p}\right|_{D^{k}\left(f_{s}, \mathcal{P}\right)}$.

We choose the coordinates such that $p\left(y_{1}, \ldots, y_{n+1}\right)=y_{1}$. We denote by $\tilde{p}: \mathbb{C}^{n+k-1} \rightarrow \mathbb{C}$ the projection onto the first coordinate. Then:

$$
D^{k}(g, \mathcal{P})=D^{k}(f, \mathcal{P}) \cap \tilde{p}^{-1}(0) .
$$

By the Lê-Greuel formula for ICIS $[4,6]$,

$$
\mu\left(D^{k}(f, \mathcal{P})\right)+\mu\left(D^{k}(g, \mathcal{P})\right)=\# \Sigma\left(\left.\tilde{p}\right|_{D^{k}\left(f_{s}, \mathcal{P}\right)}\right) .
$$

It is easy to check that $(-1)^{\operatorname{dim} D^{k}(f)} \operatorname{sign}(\mathcal{P})(-1)^{\operatorname{dim} D^{k}(f, \mathcal{P})}=1$ for any partition $\mathcal{P}$. Thus, we get:

$$
\mu_{I}(f)+\mu_{I}(g)=\sum_{k=2}^{n+1} \sum_{|\mathcal{P}|=k} \frac{\# \Sigma\left(\left.\tilde{p}\right|_{D^{k}\left(f_{s}, \mathcal{P}\right)}\right)}{\gamma(\mathcal{P})},
$$

where $\gamma(\mathcal{P})=\prod_{i} \alpha_{i} ! i^{\alpha_{i}}$.

Let $\mathcal{P}$ be a partition of $k$, if $\left|\mathcal{P}^{\prime}\right|=k$ and $\mathcal{P}^{\prime} \geq \mathcal{P}$ then any critical point of $\left.\tilde{p}\right|_{D^{k}\left(f_{s}, \mathcal{P}^{\prime}\right)}$ is a critical point of $\left.\tilde{p}\right|_{D^{k}\left(f_{s}, \mathcal{P}\right)}$. This implies

$$
\# \Sigma\left(\left.\tilde{p}\right|_{D^{k}\left(f_{s}, \mathcal{P}\right)}\right)=\sum_{\substack{\left|\mathcal{P}^{\prime}\right|=k \\ \mathcal{P}^{\prime} \geq \mathcal{P}}} \alpha\left(\mathcal{P}, \mathcal{P}^{\prime}\right) \# \Sigma\left(\left.\tilde{p}\right|_{D^{k}\left(f_{s}, \mathcal{P}^{\prime}\right)^{0}}\right),
$$

where $\alpha\left(\mathcal{P}, \mathcal{P}^{\prime}\right)$ is defined by

$$
\alpha\left(\mathcal{P}, \mathcal{P}^{\prime}\right):=\frac{\# \rho_{\mathcal{P}}^{-1}\left(\rho_{\mathcal{P}^{\prime}}(y)\right)}{\# \rho_{\mathcal{P}^{\prime}}^{-1}\left(\rho_{\mathcal{P}^{\prime}}(y)\right)}
$$

for a generic point $y$ in $D^{k}\left(f_{s}, \mathcal{P}^{\prime}\right)$. We can see $\alpha\left(\mathcal{P}, \mathcal{P}^{\prime}\right)$ as the number of times that a generic point of $D^{k}\left(f_{s}, \mathcal{P}^{\prime}\right)$ appears repeated in $D^{k}\left(f_{s}, \mathcal{P}\right)$. By 
Remark 2.6 and Lemma 2.7,

$$
\begin{aligned}
\mu_{I}(f)+\mu_{I}(g) & =\sum_{k=2}^{n+1} \sum_{|\mathcal{P}|=k} \frac{\# \Sigma\left(\left.\tilde{p}\right|_{D^{k}\left(f_{s}, \mathcal{P}\right)}\right)}{\gamma(\mathcal{P})} \\
& =\sum_{k=2}^{n+1} \sum_{|\mathcal{P}|=k} \sum_{\substack{\left|\mathcal{P}^{\prime}\right|=k \\
\mathcal{P}^{\prime} \geq \mathcal{P}}} \frac{\alpha\left(\mathcal{P}, \mathcal{P}^{\prime}\right)}{\gamma(\mathcal{P})} \# \Sigma\left(\left.\tilde{p}\right|_{\left.D^{k}\left(f_{s}, \mathcal{P}^{\prime}\right)^{0}\right)}\right. \\
& =\sum_{k=2}^{n+1} \sum_{\left|\mathcal{P}^{\prime}\right|=k}\left(\sum_{\substack{|\mathcal{P}|=k \\
\mathcal{P} \leq \mathcal{P}^{\prime}}} \frac{\# \rho_{\mathcal{P}}^{-1}\left(\rho_{\mathcal{P}^{\prime}}(y)\right)}{\gamma(\mathcal{P})}\right) \frac{\# \Sigma\left(\left.\tilde{p}\right|_{\left.D^{k}\left(f_{s}, \mathcal{P}^{\prime}\right)^{0}\right)}\right.}{\# \rho_{\mathcal{P}^{\prime}}^{-1}\left(\rho_{\mathcal{P}^{\prime}}(y)\right)} \\
& =\sum_{k=2}^{n+1} \sum_{\left|\mathcal{P}^{\prime}\right|=k} \frac{\# \Sigma\left(\left.\tilde{p}\right|_{\left.D^{k}\left(f_{s}, \mathcal{P}^{\prime}\right)^{0}\right)}\right.}{\# \rho_{\mathcal{P}^{\prime}}^{-1}\left(\rho_{\mathcal{P}^{\prime}}(y)\right)} \\
& =\sum_{k=2}^{n+1} \sum_{\left|\mathcal{P}^{\prime}\right|=k} \# \Sigma\left(\left.p\right|_{M^{k}\left(f_{s}, \mathcal{P}^{\prime}\right)}\right.
\end{aligned}
$$

which is nothing but the number of critical points of $\left.p\right|_{X_{s}}$.

\section{EXAMPLES}

In this section, we give some examples to illustrate the formulas of theorems 3.1 and 3.2 .

Example 4.1. (The singular plane curve $E_{6}$ )

Let $f(z)=\left(z^{3}, z^{4}\right)$ be the singular plane curve $E_{6}$, let $f_{s}(z)=\left(z^{3}+\right.$ $\left.s z, z^{4}+\frac{5}{4} s z^{2}\right)$ be a stabilisation of $f$, for $s \neq 0$.


Figure 1. The curve $E_{6}$ and its stabilisation for $s<0$

Let $M^{2}\left(f_{s},(1,1)\right)$ be the 0 -dimensional stratum of $X_{s}$. It is composed by three points, they correspond to three double transversal points. Let $M^{1}\left(f_{s},(1)\right)$ be the 1-dimensional stratum. If we compose $f_{s}$ with $p(z, u)=z$ there are two critical points in a neighbourhood of the origin, so $\# \sum p_{\left.\right|_{X_{s}}}=$ 5 .

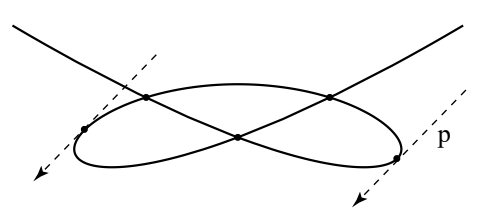

FiguRE 2. Critical points in $X_{s}$ 
Now, since the multiplicity of $f, m_{0}(f)=3$ and the image Milnor number of $f$ is $\mu_{I}(f)=3, \mu_{I}(f)+m_{0}(f)-1=5$ as predicted by the formula.

When $n>1$, we proceed in the following way: Let $f:\left(\mathbb{C}^{n}, 0\right) \rightarrow\left(\mathbb{C}^{n+1}, 0\right)$ be a corank 1 finitely determined map germ written as

$$
f(x, z)=\left(x, h_{1}(x, z), h_{2}(x, z)\right), x \in \mathbb{C}^{n-1}, z \in \mathbb{C} .
$$

Let $f_{s}$ be a stabilisation of $f$. The image of $f_{s}$ is denoted by $X_{s}$. First, we calculate the number of critical points of the restriction of $p$ to $X_{s}$, for the generic linear projection $p\left(y_{1}, \ldots, y_{n+1}\right)=y_{1}$. We separate the image set $X_{s}$ in strata of different dimensions given by stable types, which correspond to the sets $M^{k}\left(f_{s}, \mathcal{P}\right)$. The $n$-dimensional stratum, $M^{1}\left(f_{s},(1)\right)$, is composed of the regular part of $f_{s}$. So, the restriction $\left.p\right|_{M^{1}\left(f_{s}\right)}$ has not critical points.

The $(n-1)$-dimensional stratum is composed of $M^{2}\left(f_{s},(1,1)\right)$. To calculate the critical points, we will work with the inverse image by $\epsilon^{2}$, that is, $D^{2}\left(f_{s},(1,1)\right)=D^{2}\left(f_{s}\right)$. The double point space $D^{2}\left(f_{s}\right)$ is a subset of $\mathbb{C}^{n+1}$, but we take a projection of $D^{2}\left(f_{s}\right)$ in the first $n$ variables. So, we denote by $D\left(f_{s}\right)$ the projection of double point space in $\mathbb{C}^{n}$. The double point space $D\left(f_{s}\right)$ is a hypersurface in $\mathbb{C}^{n}$ given by the resultant of $P_{s}$ and $Q_{s}$ with respect to $z_{2}$, where $P_{s}=\frac{h_{1, s}\left(x, z_{2}\right)-h_{1, s}\left(x, z_{1}\right)}{z_{2}-z_{1}}$ and $Q_{s}=\frac{h_{2, s}\left(x, z_{2}\right)-h_{2, s}\left(x, z_{1}\right)}{z_{2}-z_{1}}$. This gives the defining equation of $D\left(f_{s}\right)$, denoted by $\lambda_{s}(x, z)=0$.

To calculate the critical points of the set $D\left(f_{s}\right)$ we take the linear projection $\tilde{p}\left(x_{1}, \ldots, x_{n-1}, z\right)=x_{1}$. Note that the hypersuface $D\left(f_{s}\right)$ also contains the critical points of the other $k$-dimensional strata, with $k<n-1$. Then, it will be sufficient to compute critical points here, in order to have all the critical points. We have that $\left(x_{1}, \ldots, x_{n-1}, z\right)$ is a critical point of $\tilde{p}_{\left.\right|_{D\left(f_{s}\right)}}$ if $\lambda_{s}(x, z)=0$ and $J\left(\lambda_{s}, \tilde{p}\right)(x, z)=0$, where $J\left(\lambda_{s}, \tilde{p}\right)$ is the Jacobian determinant of $\lambda$ and $\tilde{p}$.

If a critical point of $\tilde{p}_{\left.\right|_{D\left(f_{s}\right)}}$ corresponds to a $m$-multiple point, then we will have $m$ critical points in $D\left(f_{s}\right)$ for one in the image of $f_{s}$. Thus, once the critical points of each type are obtained, we have to divide by the multiplicity of the point. In this way, we obtain the number of critical points of $p$ in the image of $f_{s}$.

On the other hand, we compute separately the image Milnor numbers of $f$ and $g$ in order to check the formulas.

Example 4.2. (The germ $F_{4}$ in $\left.\mathbb{C}^{3}\right)$ Let $f(x, z)=\left(x, z^{2}, z^{5}+x^{3} z\right)$ be the germ $F_{4}$. Let $f_{s}(x, z)=\left(x, z^{2}, z^{5}+x s z^{3}+\left(x^{3}-5 x s-s\right) z\right)$ be a stabilisation of $f$, for $s \neq 0$. By [10], $f$ is a 1-parameter unfolding of the plane curve $A_{4}$, $g(z)=\left(z^{2}, z^{5}\right)$ and in fact, $g$ is the transverse slice of $f$.


Figure 3. The germ $F_{4}$ and its stabilisation for $s>0$ 
Let $M^{3}\left(f_{s},(1,1,1)\right) \cup M^{2}\left(f_{s},(2)\right)$ be the 0 -dimensional strata of $X_{s}$. In our case, there are not triple points and there are three cross caps in $M^{2}\left(f_{s},(2)\right)$.

Let $M^{2}\left(f_{s},(1,1)\right)$ be the 1-dimensional stratum of $X_{s}$. As we said, let $D^{2}\left(f_{s}\right)$ be the double point curve in $\mathbb{C}^{3}$ and by projecting in the first two coordinates, we have the double point curve in $\mathbb{C}^{2}$, denoted by $D\left(f_{s}\right)$.

We compute the resultant of $P_{s}$ and $Q_{s}$ respect to $z_{2}$, where $P_{s}$ and $Q_{s}$ are the divided differences. The double point curve of $f_{s}$ in $\mathbb{C}^{2}$ is the plane curve

$$
\lambda_{s}(x, z)=-s-5 s x+x^{3}+s x z^{2}+z^{4} .
$$

The critical points of the restriction $\left.p\right|_{D}\left(f_{s}\right)$ are given by $\lambda_{s}\left(x_{0}, z_{0}\right)=0$ and $J\left(\lambda_{s}, \tilde{p}\right)\left(x_{0}, z_{0}\right)=0$, where $\tilde{p}(x, z)=x$.
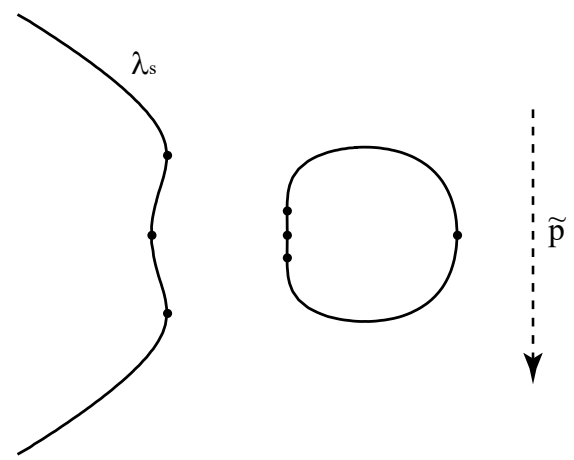

Figure 4. Cusps and tacnodes in the double point curve

Nine critical points are obtained. Three of these points are cusps in $g_{x, s}$ which correspond to the three cross caps of $f_{s}$. Then, the other six critical points in $\tilde{p}_{\left.\right|_{\lambda_{s}\left(x_{0}, z_{0}\right)=0}}$ correspond to three tacnodes in $g_{x, s}$ which are represented in the double point curve when a vertical line is tangent at two points of $D\left(f_{s}\right)$. So, each two of these critical points in $\lambda_{s}$ correspond to one tacnode of $g_{x, s}$ in $M^{2}\left(f_{s},(1,1)\right)$. Note that in the Fig. 4 there are only two tacnodes, that is because the other is a complex tacnode.

Finally, in the 2-dimensional stratum $M^{1}\left(f_{s},(1)\right)$ there are not critical points. So, the number of critical points in $X_{s}$ is $\left.\# \Sigma p\right|_{X_{s}}=6$, three cusps, three tacnodes and zero triple points. Then, $\left.\# \Sigma p\right|_{X_{s}}=C+J+T$ where $C, J, T$ are the numbers of cusps, tacnodes and triple points respectively of $g_{x, s}$. By $[10], \mu_{I}(f)=C+J+T-\delta(g)$. Since $g$ is a plane curve, we have that $\mu_{I}(g)=\delta(g)$ (see [12]). So,

$$
\left.\# \Sigma p\right|_{X_{s}}=C+J+T=\mu_{I}(f)+\mu_{I}(g) .
$$

\section{REFERENCES}

1. J. Fernández de Bobadilla, J. J. Nuño-Ballesteros, and G. Peñafort-Sanchis, A Jacobian module for disentanglements and applications to Mond's conjecture, arXiv:1604.02422, 2016.

2. Mark Goresky and Robert MacPherson, Stratified Morse theory, Ergebnisse der Mathematik und ihrer Grenzgebiete (3) [Results in Mathematics and Related Areas (3)], vol. 14, Springer-Verlag, Berlin, 1988. MR 932724

3. V. Goryunov and D. Mond, Vanishing cohomology of singularities of mappings, Compositio Mathematica 89 (1993), no. 1, 45-80. 
4. G. M. Greuel, Der Gauss-Manin-zusammenhang isolierter singularitäten von vollständigen durchschnitten, Math. Ann. 214 (1975), 235-266.

5. N. Kirk and K. Houston, On the classification and geometry of corank 1 map-germs from three-space to four-space, Singularity Theory: Proceedings of the European Singularities Conference, August 1996, Liverpool and Dedicated to CTC Wall on the Occasion of His 60th Birthday, vol. 263, Cambridge University Press, 1999, p. 325.

6. D. T. Lê, Computation of the Milnor number of an isolated singularity of a complete intersection, Funkcional. Anal. i Priložen. 8 (1974), no. 2, 45-49 (Russian).

7. I. Macdonald, Symmetric functions and hall polynomials, Oxford university press, 1998.

8. W. L. Marar, The Euler characteristic of the disentanglement of the image of a corank 1 map germ, Singularity Theory and its Applications, Lecture Notes in Math., Springer, 1991, pp. 212-220.

9. W. L. Marar and D. Mond, Multiple point schemes for corank 1 maps, J. London Math. Soc. (2) 39 (1989), no. 3, 553-567.

10. W. L. Marar and J. J. Nuño-Ballesteros, Slicing corank 1 map germs from $\mathbb{C}^{2}$ to $\mathbb{C}^{3}$, Q. J. Math. 65 (2014), no. 4, 1375-1395.

11. D. Mond, Vanishing cycles for analytic maps, Singularity theory and its applications, Part I (Coventry, 1988/1989), Lecture Notes in Math., vol. 1462, Springer, Berlin, 1991, pp. 221-234.

12. Looking at bent wires- $\mathcal{A}_{e}$-codimension and the vanishing topology of parametrized curve singularities, Math. Proc. Cambridge Philos. Soc. 117 (1995), no. 2, 213-222.

13. J. J. Nuño-Ballesteros and G. Peñafort-Sanchis, Multiple point spaces of finite holomorphic maps, arXiv:1509.04990, 2015.

Departament de Matemàtiques, Universitat de València, Campus de BurjasSOT, 46100 BURJASSOT SPAIN

E-mail address: Juan.Nuno@uv.es

E-mail address: irpato@alumni.uv.es 